\title{
Evaluation of Timed Release Calcium Sulfate (CS-TR) Bone Graft Substitutes
}

\author{
John L. Ricci,* Michael J. Weiner,* Donato Di Iorio**, Sachin Mamidwar, ${ }^{* * *}$ and Harold \\ Alexander*** \\ *Department of Biomaterials and Biomimetics, New York University College of Dentistry, 345 E. \\ $24^{\text {th }}$ St., New York, NY 10010 USA \\ **Department of Applied Sciences and Oral and Dental Diseases, Dental School, University "G. \\ d'Annunzio" of Chieti-Pescara, Chieti, Italy \\ ***Orthogen Corporation, Springfield, NJ, 07081 USA
}

In vitro and in vivo testing and development of bone graft substitute materials requires detailed analysis of bone and soft tissue response to complex multiphasic biomaterials, as well as analysis of changes in the graft materials over time. This often requires high resolution imaging of the materials and their surrounding tissues as well as microanalysis of both tissue and graft materials. We are currently testing a series of alloplastic calcium sulfate (CS) based bone graft substitutes ranging from rapidly resorbing cements and particulates to longer lasting timed release CS particulates (CS-TR) that are either coated with or are a composite with poly-L-lactic acid (PLLA).

Medical grade CS (plaster of paris) particles and cements, and two types of calcium sulfate/poly-1lactic acid (PLLA) timed-release materials (CS-TR); calcium sulfate/ PLLA composite particles, and calcium sulfate particles coated with thin layers of PLLA, were examined in a series of in vitro and in vivo studies (rabbit femur and tibia defect models) at time periods from a few days up to sixteen weeks. Faxitron high resolution x-ray, micro computed tomography (MCT), undecalcified plastic embedded histology, scanning electron microscopy in secondary electron imaging (SEI) and backscattered electron imaging (BEI) modes, x-ray microprobe (XRM), and Fourier transformed infrared (FT-IR) analysis techniques have all been used to evaluate these materials and their tissue response.

The CS materials were observed to dissolve rapidly in vitro and in vivo, from the outer surface inward at rates as high as $1 \mathrm{~mm}$ per week. They were also observed to stimulate new bone formation although bone was not observed to come in direct contact with the CS. In most animal models they dissolve completely in as little as 4 weeks, leaving behind mineral deposits in the form of concentric rings in surrounding tissue. Histologically, these deposits stain like bone mineral and often show attachment of osteoid and new bone. They were observed to act as scaffolds for new bone formation. BEI, XRM, and FT-IR show that these deposits are not immature bone or residual calcium sulfate, but are calcium phosphate deposits (Figure 1) in the form of a precipitated carbonate apatite and are very similar to bone mineral in composition. These precipitates also form in vitro when CS materials are incubated in simulated body fluids without cells present. The CS-TR materials behave in a similar fashion, but the PLLA component was observed to delay dissolution for up to 16 weeks (Figure 2) depending on amounts of PLLA used and whether the PLLA was distributed throughout the particles (as in composites) or as coatings on the outsides of the CS pellets. The CS-TR materials showed excellent biocompatibility with bone, and in contrast with the basic CS materials, persisted within the matrix of newly forming trabecular bone, for more than 16 weeks, acting as a lattice for bone formation. 
CS materials have an extensive history as resorbable alloplastic bone graft substitutes. Through a combination of in vitro and in vivo experiments, undecalcified histology, electron microscopy, bulk analysis methods, and microanalysis methods, we have been able to determine a significant mechanism that partially explains the biocompatibility and stimulation of bone formation observed at CS filled bone sites [1,2]. CS materials were observed to dissolve rapidly, produce local high concentrations of calcium ion, and cause precipitation of calcium phosphate in the form of carbonate substituted apatite. This precipitate was observed to be stable in the body fluid environment, forms in vitro in the absence of cells, and acted as an osteoconductive matrix for new bone formation in vivo. CS-TR composites and coated pellets acted in a similar way, but with controlled delays in onset of bioactivity depending on the nature and extent of the PLLA component. The CS and CS-TR materials represent a range of fully resorbable bioactive bone graft substitutes, with engineered dissolution times.

[1.] Ricci JL, et al., In: Bone Engineering, edited by JE Davies, Em² Inc., Toronto, Ont. Canada, Chapter 30, 332-344, 2000.

[2.] Orsini G, et al., J Biomed Mater Res Part B: Appl Biomater 68B: 199-208, 2004.

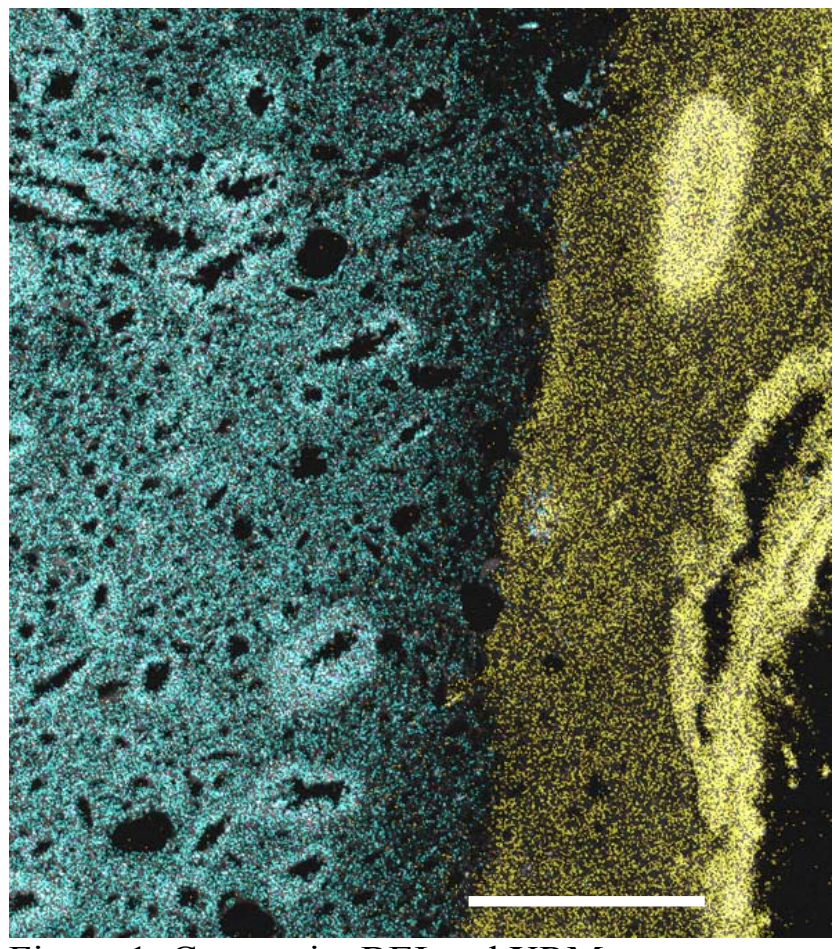

Figure 1. Composite BEI and XRM map of the edge of a CS implant in a rabbit femur after 2 weeks. Sulfur is mapped in blue and phosphorus is mapped in yellow. The yellow area represents precipitate that has formed in the in vivo environment as a result of CS dissolution. $\mathrm{Bar}=100 \mu \mathrm{m}$.

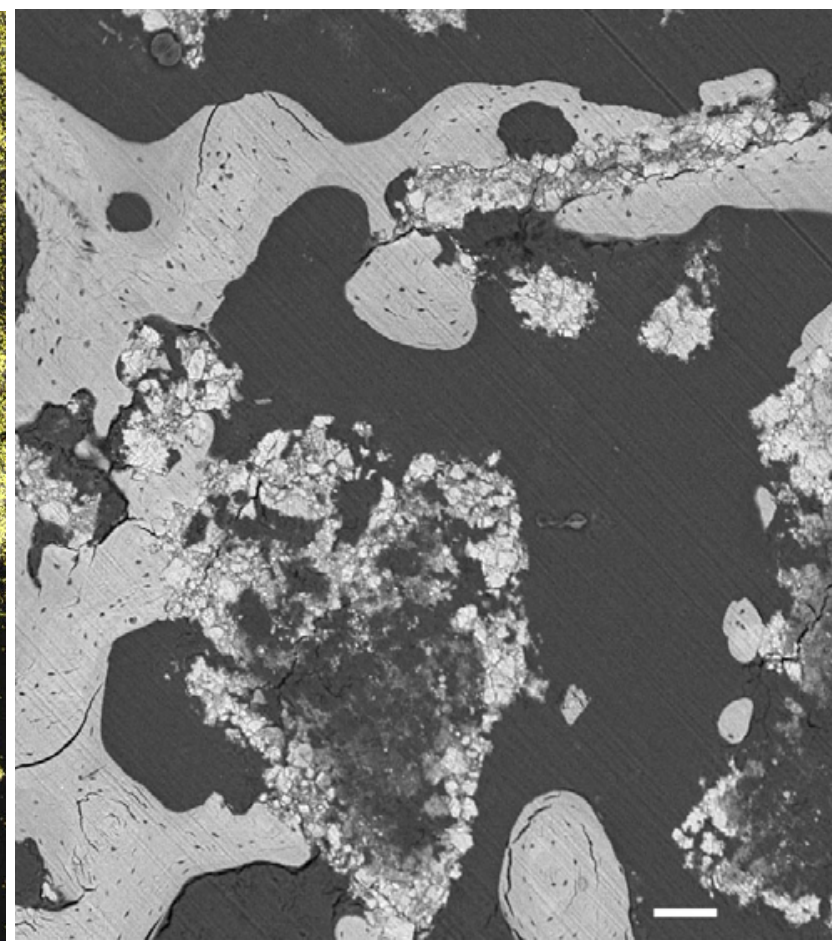

Figure 2. BEI of the remains of CS-TR pellets in a rabbit femur after 16 weeks. New bone formation is observed around clusters of calcium phosphate mineral; all that remains of the CS-TR pellets. This precipitate is more granular than the material observed at 2 weeks. Bar $=100 \mu \mathrm{m}$. 